\begin{tabular}{cc}
\hline & COMPUTATIONAL RESEARCH PROGRESS IN APPLIED SCIENCE \& ENGINEERING (CRPASE) \\
& CRPASE: TRANSACTIONS OF MECHANICAL ENGINEERING \\
\hline
\end{tabular}

\title{
Dynamic Reliability Analysis of Turbine Blades Under Combined High and Low Cycle Loadings
}

\author{
Peng Yue ${ }^{1,2}$, Bi-Sen Bai ${ }^{1}$, Juan Ma ${ }^{1,2 *}$, Xian-Dong Guo ${ }^{1}, \mathrm{He} \mathrm{Li}^{3}$ \\ ${ }^{1}$ Key Laboratory of Electronic Equipment Structure Design, Ministry of Education, Xidian University, Xi'an, 710071, P. R. China \\ ${ }^{2}$ Shaanxi Key Laboratory of Space Extreme Detection, Xidian University, Xi'an, P.R. China \\ ${ }^{3}$ Centre for Marine Technology and Ocean Engineering (CENTEC), Instituto Superior, Tecnico, Universidade de Lisboa, Lisbon, Portugal
}

\begin{tabular}{|c|c|}
\hline Keywords & Abstract \\
\hline $\begin{array}{l}\text { Combined cycle fatigue, } \\
\text { Dynamic reliability, } \\
\text { Strength degradation, } \\
\text { Turbine blade. }\end{array}$ & $\begin{array}{l}\text { Turbine blades experience complex loading interactions induced by centrifugal loads, } \\
\text { thermal loads, and vibration loads, which lead to the combined high and low cycle } \\
\text { fatigue (CCF) failure. Considering the significance in acquiring sufficient data for } \\
\text { reliability assessment, it may be imprecise to consider the load and strength as random } \\
\text { variables. To deal with the issue, material parameters and working loads involved } \\
\text { should be regarded as random variables, like high cycle fatigue stress and low cycle } \\
\text { fatigue stress as well as material strength. Hence, the dynamic reliability model with } \\
\text { strength degradation is developed based on conventional stress-strength interference } \\
\text { theory to investigate the reliability of turbine blades under CCF loading conditions. The } \\
\text { validation of the established model is performed by a numerical example, and the results } \\
\text { indicate that the proposed model considering strength deterioration is in good } \\
\text { coincidence with engineering practice. }\end{array}$ \\
\hline
\end{tabular}

\section{Introduction}

For gas turbine blades with complex structure and harsh loading environment, it is of great significance to ensure the structural integrity and operational reliability [1,2]. As one of the commonly used methods, the stress-strength interference (SSI) model is a typical tool for conducting the static reliability analysis which ignores the randomness and dynamic characteristics of applied loads and material strength over the application of loads or working time [3]. Currently, investigations on stochastic reliability problem have been performed $[4,5]$. In addition, increasing attentions have been paid for dynamic reliability analysis by considering the random uncertainness of material, applied load, environment, and production.

Generally, there exist plenty of uncertain phenomenon in engineering reliability field so that the fatigue reliability models would offer more accuracy and reasonable result with the uncertainties considered, like material parameters, applied loads, environment, and product, and so forth. Wang et al. [6] developed a time-dependent reliability model with respect to random load application for multiple times using SSI theory. Based on the previous investigations, the reliability of turbine components shown a more rational result by regarding the load and strength as random variables [7, 8]. Wang et al. [9] established a dynamic reliability model under multilevel random loads by using the moment method. Fei et al. [10] presented an extreme response surface methodbased support vector machine of regression for optimal design of dynamic reliability of turbine blade deformation. The reliability analysis of aero-engine blade was performed based on extreme response surface and finite element technique [11]. However, it is difficult to construct the reliability models of turbine blades experiencing low cycle

* Corresponding Author: Juan Ma

E-mail address: Jma@xidian.edu.cn

Received: 14 September 2021; Revised: 21 September 2021; Accepted: 23 September 2021

https://doi.org/10.52547/crpase.7.3.2393

Please cite this article as: P. Yue, B. S. Bai, J. Ma, X. D. Guo, H. Li, Dynamic Reliability Analysis of Turbine Blades Under Combined High and Low Cycle Loadings, Computational Research Progress in Applied Science \& Engineering, CRPASE: Transactions of Mechanical Engineering 7 (2021) 1-5, Article ID: 2393. 
fatigue (LCF) loads as well as high cycle fatigue (HCF) loads simultaneously, which leading to the combined high and low cycle fatigue (CCF) failure [12-14]. Accordingly, it is essential that the dynamic reliability models associated with load application times are proposed accounting for strength degradation under CCF loadings in this work.

The work is organized as follows: in section 2, dynamic reliability models of turbine blade with and without strength degradation are established by addressing the HCF loads and LCF loads simultaneously, and a numerical example for model validation and comparison of the results is performed in the subsequent section. Finally, section 4 shows the concluding remarks.

\section{The proposed Dynamic Reliability Model Under CCF Loadings}

The SSI has become one of most significant approaches to cope with mechanical reliability problems since developed by Freudenthal [15], which considering the randomness of material strength and applied load. Assuming that strength $\sigma$ and stress $S$ are independent of each other with predefined distributions, and then the components or structures would be safe when the former is larger than the latter $(\sigma>S)$, while failure will happen $(\sigma<S)$. Let the probability density functions (PDF) of strength and stress are respectively $f_{\sigma}(\sigma)$ and $f_{S}(s)$, the reliability and failure rate can be therefore expressed based on SSI theory, as

$$
\begin{aligned}
& R=P(\sigma>S)=\int_{-\infty}^{+\infty} f_{S}(s)\left[\int_{s}^{+\infty} f_{\sigma}(\sigma) d \sigma\right] d s \\
& P_{f}=P(\sigma<S)=\int_{-\infty}^{+\infty} f_{S}(s)\left[\int_{\sigma}^{s} f_{\sigma}(\sigma) d \sigma\right] d s
\end{aligned}
$$

where $f_{S}(s)$ and $f_{\sigma}(\sigma)$ are probability density functions (PDFs) of the stress and strength.

During operation, the application of random load for $m$ times is equivalent to that $m$ samples are taken from the population samples with respect to random load, denoted as $s_{1}, s_{2}, \cdots, s_{m}$. According to the maximum sequence, the reliability of components under $m$ times random load is equivalently transformed to that caused by the maximum load of the samples, as

$$
\begin{aligned}
& P\left(\sigma>s_{\max }\right) \\
& =P\left(\sigma>s_{1}, \sigma>s_{2}, \cdots, \sigma>s_{m}\right)
\end{aligned}
$$

where $s_{\max }=\max \left(s_{1}, s_{2}, \cdots, s_{m}\right)$

The cumulative distribution function (CDF) of the load is $F_{S}(s)$, and the corresponding maximum load of $m$ samples is supposed to the equivalent stress. In this analysis, the PDF of equivalent stress $X$ under the application of random load for $m$ cycles is shown as

$$
f_{X}(x)=m\left[F_{S}(x)\right]^{m-1} f_{S}(x)
$$

Accordingly, the reliability of components under the application of random load for $m$ times can be expressed by combining with Eqs. (1) and (4), as

$$
\begin{aligned}
R^{(m)} & =\int_{-\infty}^{+\infty} f_{\sigma}(\sigma) \int_{-\infty}^{\sigma} m\left[F_{S}(x)\right]^{m-1} f_{S}(x) d x d \sigma \\
& =\int_{-\infty}^{+\infty} f_{\sigma}(\sigma)\left[F_{S}(\sigma)\right]^{m} d \sigma
\end{aligned}
$$

Note that, if $m=1$, the expression of reliability in Eq. (5) reduces to the original form of the conventional SSI method. Obviously, the SSI model fails to estimate the fatigue reliability of components when the applied loads of different type is larger than one. The methodology abovementioned is insufficient in dealing with the dynamic reliability puzzles of complex mechanical components such as turbine blades, which subject to combined high and low cycle fatigue loadings $[16,17]$, as shown in Figure. 1.

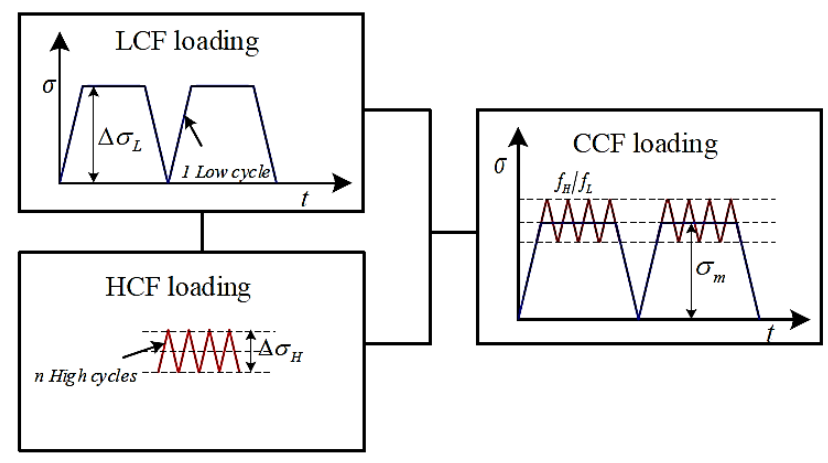

Figure 1. Load spectrum of the CCF test

As is denoted from the load spectrum, four loading parameters, including low cycle loading range $\Delta \sigma_{L}$ and its loading frequency $f_{L}$, as well as high cycle loading range $\Delta \sigma_{H}$ and the corresponding loading frequency $f_{H}$, are illustrated, in which the cyclic frequency ratio $n$ can be obtained by $n=f_{H} / f_{L}$.

Note that a LCF cycle and $n$ HCF cycles are included in one combined cycle block, and high cycle fatigue loading represented by triangular wave superimposed on the dwell stage of per one LCF loading denoted by trapezoidal wave.

For gas turbine blade, loads of the same type are independent identically distributed, such as vibration loads with high frequency and small amplitude or LCF load with low frequency and large amplitude during operation. As revealed in Figure. 1, there exist a LCF cycle and $n \mathrm{HCF}$ cycles in one combined cycle block. For HCF loads, it approximately makes the PDFs of $f_{s_{H}}\left(s_{1}\right)=f_{s_{H}}\left(s_{2}\right)=\cdots f_{s_{H}}\left(s_{n}\right)=f_{s_{H}}\left(s_{h}\right)$, then the corresponding CDFs are $F_{s_{H}}\left(s_{1}\right)=F_{s_{H}}\left(s_{2}\right)=\cdots F_{s_{H}}\left(s_{n}\right)=F_{s_{H}}\left(s_{h}\right)$. In this regard, the joint distribution function of turbine blades under $n$ type of HCF cycles can be obtained by

$$
\begin{aligned}
F_{J_{H}}\left(s_{h}\right) & =F_{s_{H}}\left(s_{1}\right) \times F_{s_{H}}\left(s_{2}\right) \times \cdots F_{s_{H}}\left(s_{n}\right) \\
& =\left[F_{s_{H}}\left(s_{h}\right)\right]^{n}
\end{aligned}
$$

Thus, the PDFs under $n$ type of HCF cycles is written as

$$
f_{J_{H}}\left(s_{h}\right)=n\left[F_{s_{H}}\left(s_{h}\right)\right]^{n-1} f_{s_{H}}\left(s_{h}\right)
$$


Reliability only involving HCF cycles of turbine blade can be obtained by the following equation

$$
\begin{aligned}
R_{H} & =\int_{-\infty}^{+\infty} f_{\sigma}(\sigma) \int_{-\infty}^{\sigma} n\left[F_{s_{H}}\left(s_{h}\right)\right]^{n-1} f_{s_{H}}\left(s_{h}\right) d s_{h} d \sigma \\
& =\int_{-\infty}^{+\infty} f_{\sigma}(\sigma)\left[F_{s_{H}}(\sigma)\right]^{n} d \sigma
\end{aligned}
$$

Assuming that major cycles of the aero-engine are independent, which suggesting that LCF loads of different types are also independent. Then the PDFs and CDFs can be represented by $f_{s_{L 1}}(s), f_{s_{L 2}}(s), \cdots, f_{s_{L N}}(s)$ and $F_{s_{L 1}}(s), \quad F_{s_{L 2}}(s), \cdots, F_{s_{L N}}(s)$, respectively.

Similarly, the joint distribution function under $N$ type of LCF cycles can be calculated by

$$
\begin{aligned}
& F_{J s_{L}}(s)=F_{s_{L 1}}(s) \times F_{s_{L 2}}(s) \times \cdots \times F_{s_{L i}}(s) \times \cdots \times F_{s_{L N}} \\
& =\prod_{i=1}^{N} F_{s_{L i}}(s)
\end{aligned}
$$

Accordingly, reliability of turbine blade under $N$ type of LCF cycles can be obtained with LCF loads considered, as

$$
R_{L}=\int_{-\infty}^{+\infty} f_{\sigma}(\sigma) \prod_{i=1}^{N} F_{s_{L i}}(\sigma) d \sigma
$$

Furthermore, assuming that high cycle vibration loads and LCF loads are independent, and the joint distribution function of turbine blade under $N$ type of combined high and low cycle fatigue loadings can be derived combined with Eqs. (6) and (9)

$$
F_{J s}(s)=\prod_{i=1}^{N}\left\{F_{s_{L i}}(s) \times\left[F_{s_{H}}\left(s_{h}\right)\right]^{n}\right\}
$$

Based on Eqs. (8), (10) and (11), the reliability of turbine blade suffering from $N$ type of CCF cycles can be shown as follows

$$
R^{(N)}=\int_{-\infty}^{+\infty} f_{\sigma}(\sigma) \prod_{i=1}^{N}\left\{F_{s_{L i}}(\sigma) \times\left[F_{s_{H}}(\sigma)\right]^{n}\right\} d \sigma
$$

In fact, the strength of the components will continue to deteriorate with the number of applied cycles during operation, and the residual strength can be expressed by [18]

$$
r(N)=\sigma_{0}[1-D(N)]^{c}
$$

where $r(N)$ is the remaining strength for $N$ combined cycle blocks, $\sigma_{0}$ is the initial strength, $c$ is the material parameter

According to the load spectrum of CCF test in Figure. 1, the combined damage of $N$ combined cycle blocks can be obtained by using linear cumulative damage rule

$D(N)=N\left(\frac{1}{N_{L C F}}+\frac{n}{N_{H C F}}\right)=N\left(\frac{1}{N_{L C F}}+\frac{n^{\prime}}{N_{L C F}}\right)$

where $n^{\prime}$ is the equivalent number of cycles under LCF loading originated from HCF vibration loads for $n$ cycles,
$N_{L C F}$ and $N_{H C F}$ are the number of cycles to failure of LCF and $\mathrm{HCF}$, respectively.

For CCF loadings, the LCF load $s_{L}$ can be considered as the mean stress $S_{m}$. Especially, the cumulative damage for $N$ combined block application times can be obtained from the perspective of the CCF loads [19]

$$
D(N)=\frac{N \int_{-\infty}^{\infty} s^{m}\left(1+n^{\prime}\right) f_{s}(s) d s}{C}
$$

where $C$ is material parameter.

Therefore, the dynamic reliability of $N$ combined cycle blocks of turbine blade considering strength degradation can be calculated by combining with Eqs. (12) - (15), as

$$
\begin{aligned}
R^{(N)} & =\int_{-\infty}^{+\infty} f_{\sigma_{0}}\left(\sigma_{0}\right) \prod_{i=0}^{N-1}\left(\int_{-\infty}^{r(N)} f_{s_{L i}}(s) d s \int_{-\infty}^{r(N)} f_{J_{S_{H}}}\left(s_{h}\right) d s_{h}\right) d \sigma_{0} \\
& =\int_{-\infty}^{+\infty} f_{\sigma_{0}}\left(\sigma_{0}\right) \prod_{i=0}^{N-1}\left(F_{S_{L i}} r(N) F_{J_{S_{H}}} r(N)\right) d \sigma_{0}
\end{aligned}
$$

According to the equivalent damage concept, the cumulative damage caused by HCF load can be transformed into total damage, and the reliability model in Eq. (12) can be further derived as one considering strength degradation, which provides a feasible and rational way for dealing with the dynamic reliability problem under CCF loadings.

\section{Numerical Example}

Aero-engine turbine blade, a complex component that generally subjects to combined high and low cycle fatigue loadings, should be taken into account in reliability modeling. Considering the uncertainness of material strength and applied CCF loads during operation, the strength, LCF loads as well as HCF loads are considered as random variables, and then the dynamic reliability with strength degradation can be calculated under multiply type of $\mathrm{CCF}$ loads by using (16), respectively.

In this section, an explosive bolt is used to validate the proposed reliability model according to the previous work $[19,20]$. Some material parameters are given by $m=2$, $a=1, C=1 e 9$. Besides, HCF load $\sigma_{H}=50 \mathrm{Mpa}$, and the variation coefficient $(\mathrm{CV})$ is taken as 0.1 due to the large uncertainty from vibration. LCF stress distribution and strength distribution are $S \sim N\left(600,20^{2}\right)$ and $\sigma \sim N(600,20)$.

In this analysis, the reliability by the proposed method with the ratio of high and low cycle stress frequency 100 and reliability based on LCF loading can be presented by using Eq (16), as shown in Figure 2. 


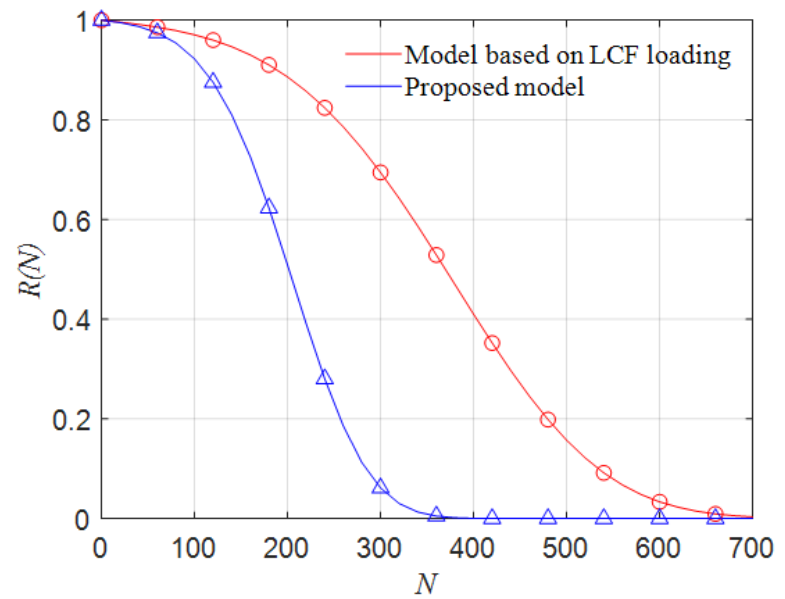

Figure 2. Comparison between models without strength degradation under different loading conditions

From Figure 2, the proposed model shows a lower reliability in contrast to that based on LCF loadings, for which it addresses the influence of CCF loadings on fatigue damage.

Moreover, reliability with different cyclic frequency ratios is illustrated to investigate the effects of HCF stress on the component under CCF loadings, as shown in Figure 3.

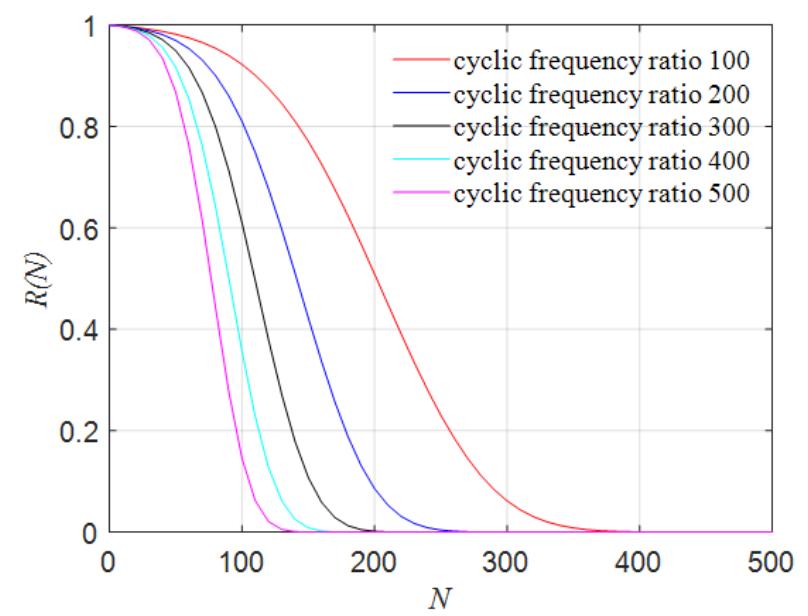

Figure 3. Comparison between models considering strength degradation under different loading conditions

It can be seen from Figure. (3), the reliability calculated by the proposed model holds a decreasing trend with the growth of high and low cycle stress frequency ratios. It also suggests that HCF loads with low amplitude and large frequency contribute considerable damage to the component. In other words, a larger cyclic frequency ratio produces a higher failure rate.

\section{Conclusion}

In this study, the dynamic reliability model with strength degradation is proposed to investigate the reliability of turbine blade based on conventional SSI method under CCF loading conditions. The uncertainties with respect to the material, CCF loads are considered under combined loading conditions, and the effects of HCF load, LCF load as well as strength deterioration on the reliability are addressed in the developed model. According to the numerical example, the reliability models under CCF loads presents an obvious effect compared to that of LCF load, and the ratio of high and low cycle stress frequency offers a considerable influence on reliability.

\section{Acknowledgment}

The authors gratefully acknowledge the support of Natural Science Foundation of China (Grant No.11572233) and Pre-Research Foundation (Grant No.61400020106) as well as the Fundamental Research Funds for the Central Universities.

\section{Conflict of Interest Statement}

The authors declare no conflict of interest.

\section{References}

[1] F. J. Golrokh, H. Ahmar, A comparison of machine learning clustering algorithms based on the DEA optimization approach for pharmaceutical companies in developing countries. Eng Transactions (2020) 1-8.

[2] H. Li, , A. P. Teixeira, C. Guedes Soares, A two-stage Failure Mode and Effect Analysis of offshore wind turbines. Renewable Energy 162 (2020) 1438-1461.

[3] H. Li, C.Guedes Soares, H. Z. Huang, Reliability analysis of floating offshore wind turbine using Bayesian Networks. Ocean Engineering 217 (2020) 107827.

[4] S. M. Hosseinian, V.N.M. Gilani, Analysis of factors affecting urban road accidents in rasht metropolis, Eng Transactions 1 (2020) $1-4$.

[5] H. Li, H.Z. Huang, Y.F. Li, J. Zhou, Physics of failure-based reliability prediction of turbine blades using multi-source information fusion. Applied Soft Computing 72 (2018) 624 635.

[6] Z. Wang, L.Y. Xie, B. Li, Time-dependent reliability model of component under random load. Chinese Journal of Mechanical Engineering 3 (2007) 20-25.

[7] H. Li, H. Díaz, C. Guedes Soares, A failure analysis of floating offshore wind turbines using AHP-FMEA methodology. Ocean Engineering 234 (2021) 109261.

[8] S.P. Zhu, Q. Liu, J. Zhou, Z.Y. Yu, Fatigue reliability assessment of turbine discs under multi-source uncertainties. Fatigue \& Fracture of Engineering Materials \& Structures 41 (2018) 1291-1305.

[9] Z.G. Wang, Model of fatigue dynamic reliability of mechanical component under multi-stage random loads. Machine design and research 3 (2014) 83-86.

[10] C. W. Fei, W. Z. Tang, \& G. C. Bai, Novel method and model for dynamic reliability optimal design of turbine blade deformation. Aerospace Science \& Technology 39 (2014) 588-595.

[11] C.Y. Zhang, C. Lu, \& C. W. Fei,. Reliability analysis of aeroengine blade based on extremum response surface method. Journal of Harbin University of Science and Technology 20 (2015) 5-10.

[12] L. Han, D.W. Huang, X.J. Yan, C. Chen, X.Y. Zhang, M.J. Qi, Combined high and low cycle fatigue life prediction model based on damage mechanics and its application in determining the aluminized location of turbine blade. International Journal of Fatigue 127 (2019) 120-130.

[13] D. Priyanka, J.N. Andrew. Aeromechanical modeling of rotating fan blades to investigate high-cycle and low-cycle fatigue interaction. Journal of Engineering for Gas Turbines and Power 137 (2015) 1-11. 
[14] S.P. Zhu, P. Yue, Z.Y. Yu, Q.Y. Wang, A combined high and low cycle fatigue model for life prediction of turbine blades. Materials 10 (2017) 698.

[15] A. M. Freudenthal, The safety of Structures. Transactions of the American Society of Civil Engineers 112 (1974) 125-159.

[16] P. Yue, J. Ma, T. Li, Ch. Zhou, H. Jiang, Fatigue life prediction based on nonlinear fatigue accumulation damage model under combined cycle loadings. Computational Research Progress in Applied Science and Engineering 6 (2020) 197-202.

[17] Yue Peng, Ma Juan, Huang Han, Shi Yang, Zu W Jean. 2021. Threshold damage-based fatigue life prediction of turbine blades under combined high and low cycle fatigue. International Journal of Fatigue150 (2021) 106323.
[18] P. Gao, L. Xie, Dynamic Reliability Analysis Method of Degraded Mechanical Components Based on Process Probability Density Function of Stress. Mathematical Problems in Engineering (2014) 1-12.

[19] P. Yue, J. Ma, C.H. Zhou, H. Jiang, P. Wriggers, A fatigue damage accumulation model for reliability analysis of engine components under combined cycle loadings. Fatigue \& Fracture of Engineering Materials \& Structures 43 (2021) 113.

[20] Yue Peng, Ma Juan, Zhou Changhu, Zu J Wean, Shi Baoquan. Dynamic fatigue reliability analysis of turbine blades under the combined high and low cycle loadings. International Journal of Damage Mechanics 30 (2021) 825-844. 